\title{
Spin injection through energy-band symmetry matching with high spin polarization in atomically controlled ferromagnet/ferromagnet/ semiconductor structures
}

\author{
Michihiro Yamada ${ }^{1}$, Fumiaki Kuroda ${ }^{2}$, Makoto Tsukahara', Shinya Yamada ${ }^{1}$, Tetsuya Fukushima ${ }^{1,3,4}$, Kentarou Sawano ${ }^{5}$, \\ Tamio Oguchi ${ }^{1,6,7}$ and Kohei Hamaya ${ }^{1}$
}

\begin{abstract}
Electrical injection of spin-polarized electrons from ferromagnets into semiconductors has been generally demonstrated through a tunneling process with insulator barrier layers that can dominate the device performance, including the electric power at the electrodes. Here, we show an efficient spin injection technique for a semiconductor using an atomically controlled ferromagnet/ferromagnet/semiconductor heterostructure with low-resistive Schottkytunnel barriers. On the basis of symmetry matching of the electronic bands between the top highly spin-polarized ferromagnet and the semiconductor, the magnitude of the spin signals in lateral spin-valve devices can be enhanced by up to one order of magnitude compared to those obtained with conventional ferromagnet/semiconductor structures. This approach provides a new solution for the simultaneous achievement of highly efficient spin injection and low electric power at the electrodes in semiconductor devices, leading to novel semiconductor spintronic architectures at room temperature.
\end{abstract}

\section{Introduction}

For the achievement of novel semiconductor (SC) architectures based on the electron spin degree of freedom, electrical injection of spin-polarized electrons from a ferromagnetic material (FM), manipulation of the injected spins by applying magnetic fields, and electrical detection of the manipulated spins have been demonstrated in SC device structures ${ }^{1,2}$. The recent progress in the observation of reliable spin transport at room temperature in group IV SC device structures is beginning to open up a way toward SC spintronic applications because of the compatibility with silicon $(\mathrm{Si})$ complementary

\footnotetext{
Correspondence: Kohei Hamaya (hamaya@ee.es.osaka-u.ac.jp)

${ }^{1}$ Center for Spintronics Research Network, Graduate School of Engineering Science, Osaka University, 1-3 Machikaneyama, Toyonaka 560-8531, Japan

${ }^{2}$ Department of Material Engineering Science, Graduate School of Engineering Science, Osaka University, 1-3 Machikaneyama, Toyonaka 560-8531, Japan

Full list of author information is available at the end of the article
}

metal-oxide-semiconductor (CMOS) technologies ${ }^{3,4}$. For high-performance SC spintronic devices in particular, the generation of large spin accumulation in SCs at room temperature is the most important issue. To date, the introduction of tunnel barriers between FMs and SCs has been generally proposed to eliminate the large conductivity mismatch between FMs and $\mathrm{SCs}^{5,6}$. High output voltages derived from the large spin accumulation in $\mathrm{Si}$ were recently demonstrated in $\mathrm{FM} / \mathrm{MgO} / \mathrm{Si}$ lateral devices at room temperature ${ }^{7,8}$. If a two-terminal magnetoresistance (MR) ratio, which is the most important performance measure of a nonvolatile memory, of more than $100 \%$ can be obtained at room temperature even in metaloxide-semiconductor field-effect-transistor structures, then one can effectively utilize the spin-related phenomena in high-performance applications of SC architectures ${ }^{9}$. However, insulator tunnel barriers, such as $\mathrm{MgO}$, cause a high parasitic resistance at the source 
and/or drain contacts, inducing a decrease in the MR ratio and the use of a high electric power in proposed SC spintronic devices ${ }^{10-12}$.

In this article, to simultaneously achieve highly efficient spin injection at room temperature and low contact resistance in SC devices, we present an efficient spin injection technique using an FM-FM-SC heterostructure, where the top FM electrode is a ferromagnetic Heusler alloy, $\mathrm{Co}_{2} \mathrm{FeAl}_{0.5} \mathrm{Si}_{0.5}$ (CFAS), the middle $\mathrm{FM}$ is atomically controlled iron (Fe) layers, and the $\mathrm{SC}$ is germanium (Ge), which not only is a next-generation channel material for CMOS but also can be applied to photonics $^{13}$ and quantum computing ${ }^{14}$. Although we have thus far developed growth techniques for singlecrystalline CFAS layers on $\mathrm{Ge}^{15}$ and Schottky-tunnel barrier electrodes with low values of the resistance area product $(R A)$ for Ge spintronic devices ${ }^{16}$, the twoterminal $M R$ ratio at room temperature has been quite low $(\leq 0.001 \%)$ because of the low spin accumulation in Ge-based lateral spin-valve (LSV) devices ${ }^{17}$. When five-six atomic layers of $\mathrm{Fe}\left(\mathrm{Fe}_{5-6}\right)$ are inserted between CFAS and Ge, we experimentally demonstrate salient enhancements in spin signals by more than one order of magnitude compared to those in the previous report on $\mathrm{Ge}^{17}$, in the LSV devices. From the structural analyses, atomically controlled $\mathrm{CFAS} / \mathrm{Fe}_{5-6} / \mathrm{Ge}(111)$, i.e., $\mathrm{FM} / \mathrm{FM} /$ SC heterostructures, can be demonstrated without inducing out-diffusion of Ge atoms into FM layers. On the basis of previous theories ${ }^{18,19}$, the transmittance of the spin-dependent conductance for the $\mathrm{CFAS} / \mathrm{Fe}_{5-6} / \mathrm{Ge}$ heterostructure is examined along the [111] direction. As a result, we theoretically find that the CFAS/ $/ \mathrm{Fe}_{5-6} / \mathrm{Ge}$ heterostructure along the $<111>$ direction has energyband symmetry matching, leading to efficient injection of spin-polarized electrons into the conduction band of Ge. In the CFAS/ $/ \mathrm{Fe}_{5-6} / \mathrm{Ge}$ LSV devices, we can experimentally achieve a more than one order of magnitude enhancement in the MR ratio $(\sim 0.04 \%)$ at room temperature and the lowest electric power $(\sim 0.12 \mathrm{~mW})$ for obtaining a high MR ratio. Given these novel findings, we can conclude that spin injection through symmetry matching of the electronic bands between the top highly spin-polarized FM and SC in FM/FM/SC heterostructures provides a new solution for the simultaneous achievement of highly efficient spin injection at room temperature and low electric power at the electrodes, leading to novel SC spintronic architectures.

\section{Results}

Effect of Fe atomic layer insertion on spin signals

To examine the effect of Fe atomic layer insertion on spin transport in SC-based spintronic devices, we prepared LSV devices with an $n$-Ge spin-transport channel and two ferromagnetic contacts, as shown in Fig. 1a. The detailed fabrication procedure of the LSV devices is described in the Materials and methods. Additionally, similar LSV structures were presented in our previous works ${ }^{15-17,20,21}$. We note that the highly spin-polarized material CFAS is utilized as a spin injector and detector for Ge, where CFAS is one of the ferromagnetic Heusler a

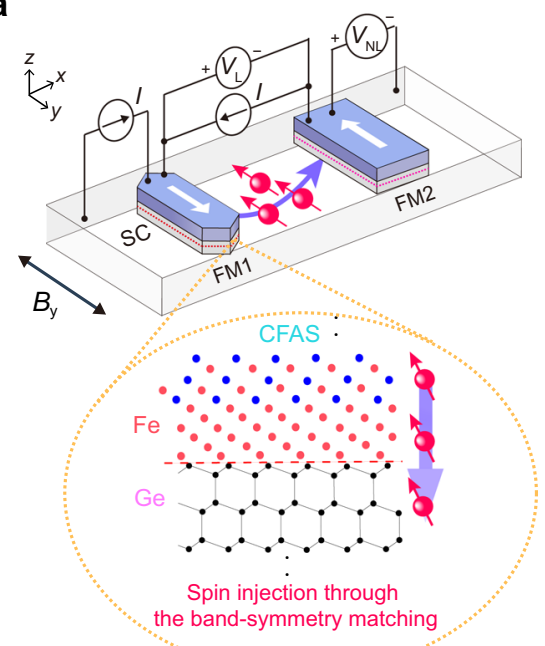

b

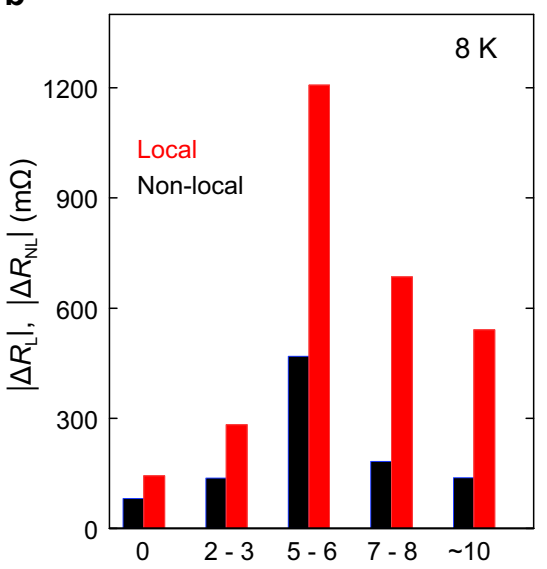

The number of inserted $\mathrm{Fe}$ atomic layers c

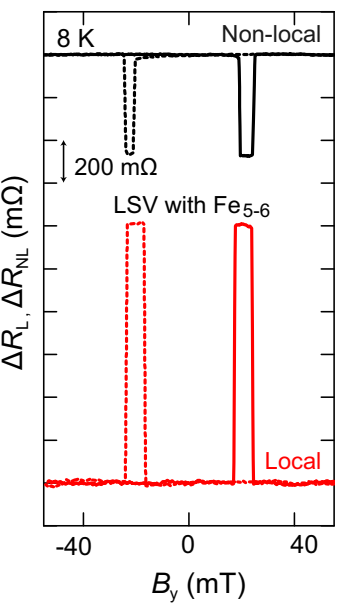

Fig. 1 Enhancement of spin signals based on a new concept. a Schematic of the fabricated FM-SC-FM lateral spin-valve (LSV) device, in which the terminal configurations of nonlocal and local voltage measurements are drawn. The enlarged schematic illustration shows the concept of spin injection through energy-band symmetry matching between $\mathrm{CO}_{2} \mathrm{FeAl}_{0.5} \mathrm{Si}_{0.5}$ (CFAS) and Ge with atomically controlled Fe layers. b Variation in the nonlocal and local spin signals $\left(\triangle R_{\mathrm{NL}}\right.$ and $\left.\Delta R_{\mathrm{L}}\right)$ with the number of inserted Fe atomic layers at $8 \mathrm{~K}$. c Nonlocal and local magnetoresistance curves measured at $8 \mathrm{~K}$ and $I=-0.5 \mathrm{~mA}$ for the LSV device with five-six atomic layers of Fe (LSV with Fe $\mathrm{Fe}_{5-6}$ ). 
alloys $^{15,16,22}$. To verify the effect of $\mathrm{Fe}$ atomic layer insertion on spin injection and detection, nonlocal and local voltage measurements were carried out on all LSV devices with insertion of various $\mathrm{Fe}$ atomic layers in the four- and two-terminal configurations ${ }^{23-26}$, respectively. In Fig. 1b, the magnitude of the nonlocal $\left(\Delta R_{\mathrm{NL}}=\Delta V_{\mathrm{NL}} /\right.$ $I)$ and local $\left(\Delta R_{\mathrm{L}}=\Delta V_{\mathrm{L}} / I\right)$ spin signals at $8 \mathrm{~K}$ as a function of the number of inserted $\mathrm{Fe}$ atomic layers is summarized, where representative nonlocal and local spin signals for the LSV device with $\mathrm{Fe}_{5-6}$ at $8 \mathrm{~K}$ are also shown in Fig. 1c. Here, the plotted values of $\left|\Delta R_{\mathrm{NL}}\right|$ and $\left|\Delta R_{\mathrm{L}}\right|$ are the maximum values observed in this study, and the value of $\mid$ $\Delta R_{\mathrm{L}} \mid$ includes the enhancement effect due to the nonlinear electrical spin conversion at a biased FM/SC contact $^{21,27}$. According to Fig. 1b, salient enhancements in both $\left|\Delta R_{\mathrm{NL}}\right|$ and $\left|\Delta R_{\mathrm{L}}\right|$ can be observed for the LSV with $\mathrm{Fe}_{5-6}$. With further increases in the number of inserted $\mathrm{Fe}$ atomic layers above $\mathrm{Fe}_{5-6},\left|\Delta R_{\mathrm{NL}}\right|$ and $\left|\Delta R_{\mathrm{L}}\right|$ gradually decrease. Since this feature is quite important, we will discuss this point later. In this study, the tendencies observed in Fig. 1b are reproduced for many LSV devices.

The value of $R A$ for the spin injection and detection electrodes was maintained at less than $0.40 \mathrm{k} \Omega \mu \mathrm{m}^{2}$ after $\mathrm{Fe}$ atomic layer insertion because the electrical characteristics of the electrodes were controlled by the phosphorus $\delta$-doped layer near the CFAS/Ge interface ${ }^{16,20}$. The detailed $J-V$ characteristics are presented in Supplementary Fig. S1. In addition, from the Hanle effect analysis based on the one-dimensional spin drift-diffusion model $^{1,16,24-26}$, we confirmed that the spin transport properties, such as the spin diffusion length $\left(\lambda_{\mathrm{N}}\right)$, in the used Ge channel were almost equivalent $\left(\lambda_{\mathrm{N}}=0.84 \pm\right.$ $0.07 \mu \mathrm{m})$ for all the LSV devices, as shown in Supplementary Fig. S2. For these reasons, the one-order enhancements in $\left|\Delta R_{\mathrm{NL}}\right|$ and $\left|\Delta R_{\mathrm{L}}\right|$ shown in Fig. $1 \mathrm{~b}$ are attributed to the insertion of the controlled Fe atomic layers between CFAS and Ge.

Using the standard theories of LSV devices ${ }^{6}$ and the extracted parameters of the used $n$-Ge at $8 \mathrm{~K}$ (Supplementary Figs. S1 and S2), we can tentatively estimate the spin injection/detection efficiency $(P)$ and interface spin polarization $(\gamma)$ from the nonlocal and local spin signals, respectively. The detailed methods for the definition and estimation of $P$ and $\gamma$ are described in the Materials and methods. Figure 2 displays $P$ (left axis) and $\gamma$ (right axis) as a function of the number of inserted Fe atomic layers at $8 \mathrm{~K}$. Both values evidently show a peak, reaching $P \sim 0.25$ and $\gamma \sim 0.28$, for the LSV with $\mathrm{Fe}_{5-6}$. With further increases in the number of inserted Fe layers to more than seven atomic layers, the values of $P$ and $\gamma$ gradually decrease. These data in Figs. $1 \mathrm{~b}$ and 2 reveal that the ability for spin injection and detection in SC-based LSV devices can be controlled by adjusting the number of $\mathrm{Fe}$ atomic layers inserted between CFAS and Ge.

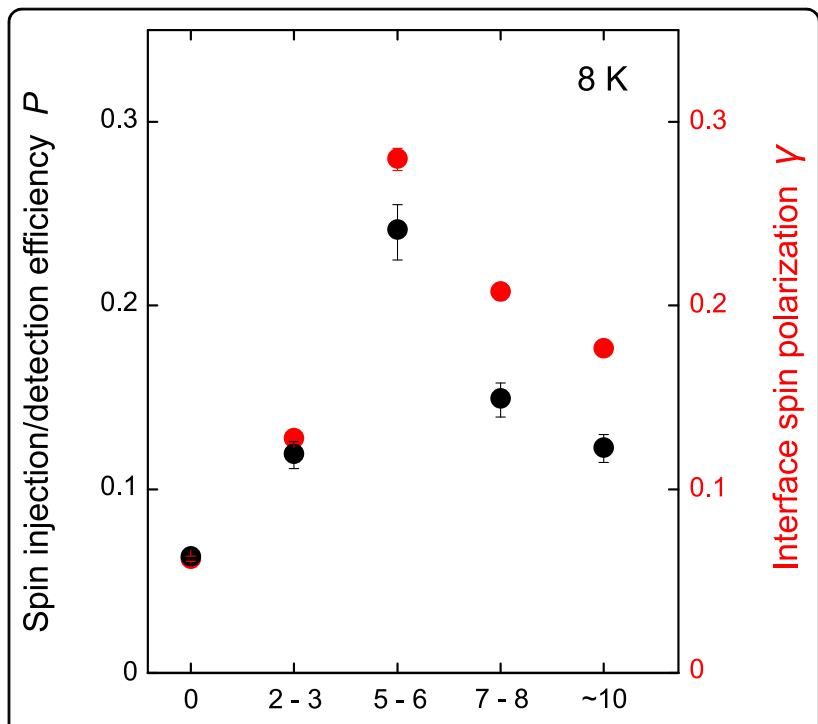

The number of inserted $\mathrm{Fe}$ atomic layers

Fig. 2 Enhanced spin injection/detection efficiency and interface spin polarization. Inserted $\mathrm{Fe}$ atomic layer dependence of the spin injection/detection efficiency $(P)$ and interface spin polarization $(\gamma)$ at $8 \mathrm{~K}$. These values were estimated from the one-dimensional spin diffusion model, shown in the Materials and methods.

\section{Structural and elemental analyses for the spin injector}

To characterize the CFAS/Fe $/ \mathrm{F}_{-6} / \mathrm{Ge}$ interface structure, high-angle annular dark field (HAADF) scanning transmission electron microscopy (STEM) imaging and selected area electron diffraction (SAED) were performed. Figure 3a shows a low-magnification HAADF-STEM image. Uniform CFAS and phosphorus $\delta$-doped Ge lay$\operatorname{ers}^{16}$ can be observed, and there is a bright layer indicating $\mathrm{Fe}_{5-6}$ between CFAS and Ge, where the contrast in the HAADF-STEM image gives information on the mass distribution in the matrix and is related to the atomic number of each element $\left(Z^{2}\right)$. Thus far, due to the outdiffusion of Ge atoms, we have observed mass contrast fluctuations in the CFAS layer near the CFAS/Ge interface for the LSV devices with no Fe insertion ${ }^{20}$. In contrast, Fig. 3a clearly shows an improvement in the quality of the CFAS/Ge interface, in particular, the composition fluctuation in the CFAS layer. Thus, the insertion of $\mathrm{Fe}$ atomic layers between CFAS and Ge is quite effective in improving the interface quality.

The cube-on-cube epitaxy between CFAS and Ge was confirmed by the SAED pattern [Fig. 3b], leading to an overlap of the diffraction reflections between them ${ }^{28}$. As a result, the epitaxial relationship between CFAS and Ge was determined to be CFAS(111)||Ge(111) and CFAS(11)0)||Ge( $\overline{1} 10)$, similar to in previous work $^{28}$. Even after Fe atomic layer insertion, the epitaxial relationship between CFAS and Ge is maintained. We also performed atomic-resolution HAADF-STEM imaging at the CFAS/ $/ e_{5-6} /$ Ge interface, as 

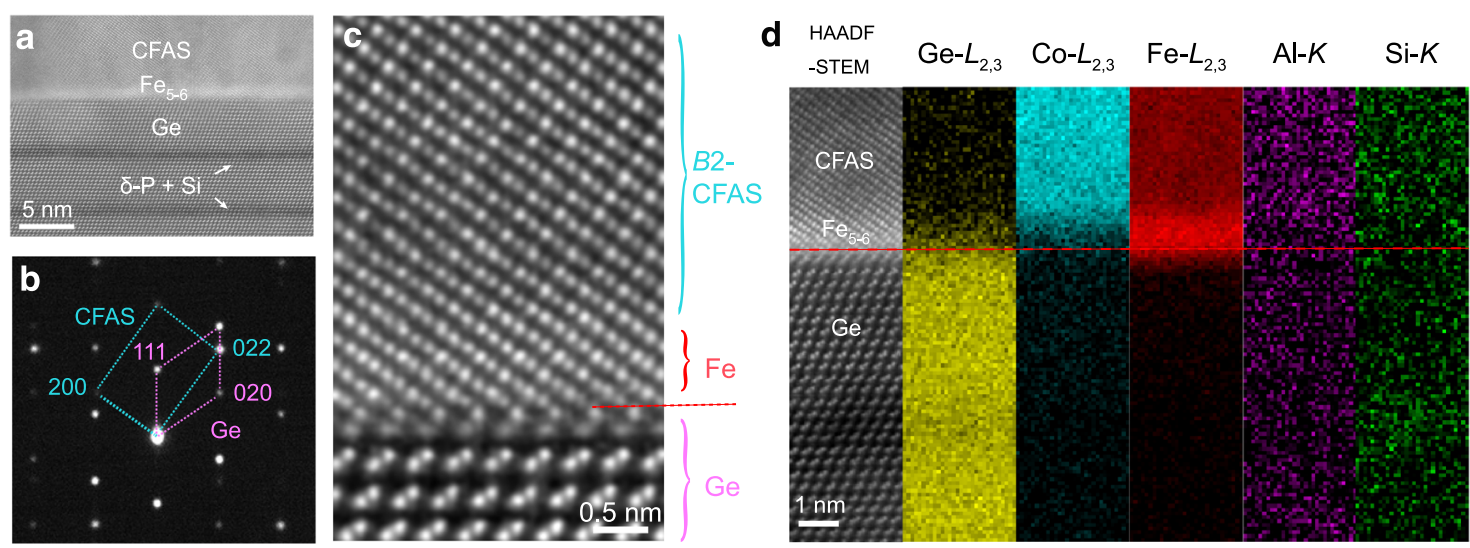

Fig. 3 Atomically controlled $\mathrm{Co}_{2} \mathrm{FeAl}_{0.5} \mathrm{Si}_{0.5} / \mathrm{Fe}_{5-6} / \mathrm{Ge}$ interface. a Low-magnification high-angle annular dark field (HAADF) scanning transmission electron microscopy (STEM) image. A uniform interface and two $\delta$-doped layers $(\delta-\mathrm{P}+\mathrm{Si})$ can be observed. b Selected area electron diffraction (SAED) pattern from an area including $\mathrm{CO}_{2} \mathrm{FeAl}_{0.5} \mathrm{Si}_{0.5}$ (CFAS), $\mathrm{Fe}_{5-6}$, and Ge. The unit cell of the diffraction pattern is labeled with a blue dashed rectangle for the CFAS layer and a red dashed rhombus for the Ge layer. $\mathbf{c}$ High-resolution HAADF-STEM image at the CFAS/Fe ${ }_{5-6} / \mathrm{Ge}$ interface. $\mathbf{d}$ EELS mappings showing the intensities of $\mathrm{Ge}-L_{2,3}, \mathrm{Co}-L_{2,3}, \mathrm{Fe}-L_{2,3}, \mathrm{Al}-K$, and $\mathrm{Si}-K$ edge signals together with the HAADF-STEM image obtained simultaneously.

shown in Fig. 3c. On top of the Ge surface, high-intensity atomic columns with a thicknesses of $0.7-0.8 \mathrm{~nm}$, implying the presence of $\mathrm{Fe}_{5-6}$, can be observed. After the top of the inserted $\mathrm{Fe}$ atomic layers, alternating Co (brighter) and $\mathrm{Fe}-\mathrm{Si} / \mathrm{Al}$ (darker) atomic columns, are distinctively observed, which means that a $B 2$-ordered CFAS layer is near the interface. To determine the chemical distribution of constituent elements near the interface, electron energy loss spectroscopy mapping was performed, and the result for the $\mathrm{Fe}_{5-6}$ interface is displayed in Fig. 3d. An evident increase in the intensity of the Fe- $L_{2,3}$ edge spectrum is observed from the mapping between CFAS and Ge. Additionally, out-diffusion of the Ge atoms is clearly suppressed compared to our previous work ${ }^{20}$. Therefore, the insertion of the Fe atomic layers between CFAS and Ge also enables the formation of $B 2$-ordered CFAS near the interface by suppressing the composition fluctuation due to the outdiffusion of Ge atoms ${ }^{20,28}$.

From structural and elemental analyses near the CFAS/ $\mathrm{Fe}_{5-6} / \mathrm{Ge}$ interface, we can conclude that the enhancement in $\left|\Delta R_{\mathrm{NL}}\right|$ and $\left|\Delta R_{\mathrm{L}}\right|$ in Fig. 1b originates partly from the suppression of the composition fluctuation in the CFAS layer. Additionally, in our previous work ${ }^{16}$, we confirmed that the absence of the CFAS layer leads to inefficient spin injection into Ge. Thus, we infer that experimentally, the formation of $B 2$-ordered CFAS layers at the FM/SC electrodes improved the values of $P$ and $\gamma$ in Fig. 2. However, from first-principles density functional theory (DFT) calculations, we have obtained that the electronic band structure of the Fe-inserted CFAS/Ge interface is not expected to evolve a highly spin polarized nature, as shown in Supplementary Fig. S3. This means that the experimental enhancements in $P$ and $\gamma$ in Fig. 2 are not caused directly by the improvement in the spin polarization at the CFAS/Ge interface due to the insertion of atomically controlled $\mathrm{Fe}$ layers. Recently, Rashba coupling-induced efficient spin-tocharge conversion at the $\mathrm{Fe} / \mathrm{Ge}(111)$ interface was proposed $^{29}$. We may regard this mechanism as one of the possible origins for the enhancements in $P$ and $\gamma$ when inserting Fe atomic layers between CFAS and Ge. However, because there is a clear correlation among the number of inserted Fe atomic layers, $\left|\Delta R_{\mathrm{NL}}\right|,\left|\Delta R_{\mathrm{L}}\right|, P$, and $\gamma$, the present results cannot be explained only by efficient spin injection based on the improvement due to the Rashba coupling-induced efficient spin-to-charge conversion discussed in ref. ${ }^{29}$.

\section{Discussion}

Energy-band symmetry and spin-dependent transmittance

Considering Fig. 1a, we hereinafter focus on the spindependent transport properties of electrons based on the band symmetry matching arguments for $\mathrm{CFAS} / \mathrm{Fe} / \mathrm{Ge}$ heterostructures. On the basis of first-principles DFT calculations $^{30}$, we evaluate the energy bands, spindependent transmittance, and spin polarization in $\mathrm{CFAS} / \mathrm{Fe} / \mathrm{Ge} / \mathrm{Fe} / \mathrm{CFAS}$ heterojunctions. Computational details and calculation models are described in the Materials and methods. Figure 4 displays theoretically calculated bulk energy bands of CFAS, Fe, and Ge along the [111] direction. Although the value of the band gap for Ge is underestimated in our calculation conditions with the generalized gradient approximation (GGA), the conduction band symmetry can be precisely depicted. According to Fig. 4c, we first find that the conduction bands of Ge near the Fermi level $(E=0)$ have $\Lambda_{1}$ symmetry, which is the identity representation of $C_{3 \mathrm{v}}$ 

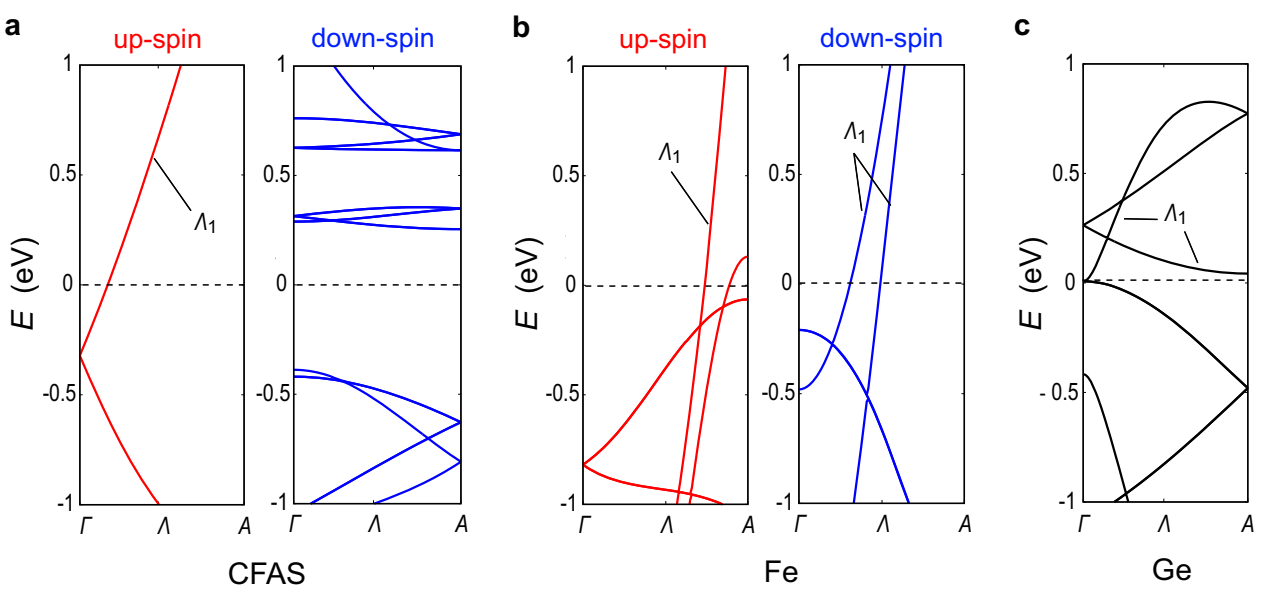

Fig. 4 Energy bands along the [111] direction. a-c Bulk energy bands of $\mathrm{CO}_{2} \mathrm{FeAl}_{0.5} \mathrm{Si}_{0.5}$ (CFAS), $\mathrm{Fe}$, and $\mathrm{Ge}$, respectively, along the [111] direction. All the energy-band structures were depicted by using the $\mathrm{k} / /[001]$ direction of hexagonal unit cells because of the hexagonal atomic arrangement of the fcc structure along the [111] direction. When the generalized gradient approximation (GGA) ${ }^{30}$ was adopted for the exchange and correlation functional, the band structures of Ge (diamond) became similar to those of a semimetal with some band crossings at the Fermi level around the $\Gamma$ point. The $\Gamma$ and A points of the hexagonal Brillouin zone considered here correspond to the $\Gamma$ and $L$ points of the fcc Brillouin zone, respectively. The conduction bands of Ge near the Fermi level $(E=0)$ have $\Lambda_{1}$ symmetry.

symmetry. This means that the $\Lambda_{1}$ symmetry bands of the used FM electrodes can play a crucial role in the efficient injection of spin-polarized electrons into Ge. It should be noted that for Fe in Fig. 4b, because $\Lambda_{1}$ bands at the Fermi level exist for both up-spin and down-spin electrons, which has already been mentioned in a previous theoretical work ${ }^{19}$, the Fe electrode is found to be an inefficient spin injector for Ge along the [111] direction. In Fig. 4a, on the other hand, only the $\Lambda_{1}$ band for the up-spin electrons of CFAS crosses the Fermi level, and no band for the down-spin electrons touches the Fermi level. At other $k$-points of the down-spin electrons, only small pockets are found (not shown here). Thus, the CFAS electrode is expected to be a nearly half-metallic spin injector within the GGA. Considering the energy-band symmetry along the [111] direction between CFAS and $\mathrm{Ge}$, we can conclude that the CFAS electrode is expected to be a highly efficient spin injector for Ge. Actually, the $\Lambda_{1}$ band for the up-spin electrons of CFAS has a wide energy dispersion, resulting in widespread wave functions in real space, while the down-spin electrons around the Fermi level have narrow dispersions, causing localized wave functions in real space. Since the dispersive $\Lambda_{1}$ band is dominated by $s, p_{z}$, and $d_{z}{ }^{2}$ orbitals spreading toward the [111] direction, the electrons with light effective electron mass of incident Bloch states can strongly contribute to the spin-dependent conductance in tunneling and giant MR junctions ${ }^{31,32}$.

Figures $5 \mathrm{a}$ and $\mathrm{b}$ show the optimized interfaces of $\mathrm{CFAS} / \mathrm{Ge}$ and $\mathrm{CFAS} / \mathrm{Fe}_{5} / \mathrm{Ge}$ in the heterojunctions, respectively. From the $k$-point-resolved transmittance in Fig. 5c for CFAS/Ge junctions, we can see a strong ring- shaped peak of the transmittance from the up-spin electrons around the $\Gamma$ point, whereas the transmittance from the down-spin electrons is much smaller. This feature originates from the Fermi-surface asymmetry, including the $\Lambda_{1}$ band symmetry matching of the up-spin electrons, described above. Since the energy-band symmetry leads to coupling of incident Bloch states from the CFAS electrode into $\mathrm{Ge}$, we can infer that the observed strong ring-shaped peak in Fig. $5 \mathrm{c}$ is derived from the $\Lambda_{1}$ band electrons. Here, since the $k$-point-averaged transmittance values from the up-spin electrons $\left(G_{\text {up }}\right)$ and the down-spin electrons $\left(G_{\text {down }}\right)$ are $7.9 \times 10^{-2}$ and $2.3 \times 10^{-4}$, respectively, the calculated spin polarization value $P_{\text {cal }}$ is approximately 1.0. Thus, if the ideal CFAS/Ge/CFAS junctions along the $<111>$ directions for electron transport are formed, then fully spin-polarized electrons can theoretically flow based on $\Lambda_{1}$ band symmetry matching. In Fig. 5d, for $\mathrm{CFAS} / \mathrm{Fe}_{5} / \mathrm{Ge}$ junctions, the $k$-pointresolved transmittance also shows a ring-shaped peak around the $\Gamma$ point, and the $G_{\text {up }}$ and $G_{\text {down }}$ values are $1.1 \times 10^{-1}$ and $9.8 \times 10^{-4}$, respectively. Since coupling of the incident Bloch states from the CFAS electrode through $\mathrm{Fe}_{5}$ into Ge is also derived from the $\Lambda_{1}$ band symmetry matching, the highly spin-polarized current can be protected. Due to the itinerant character in $\mathrm{Fe}$, the values of $G_{\text {up }}$ and $G_{\text {down }}$ are slightly higher than those for CFAS/Ge junctions, also leading to $P_{\text {cal }} \sim 1.0$. That is, despite the presence of the inserted Fe layers, highly spin-polarized currents can flow in CFAS/ $/ \mathrm{Fe}_{5} / \mathrm{Ge}$ heterostructures due to the Fermi-surface asymmetry, including the $\Lambda_{1}$ band symmetry matching of the up-spin electrons. 
a

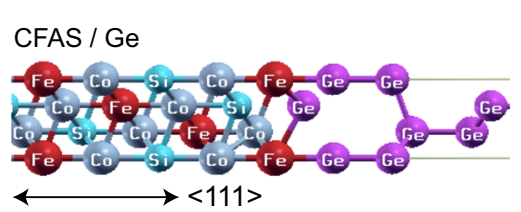

b

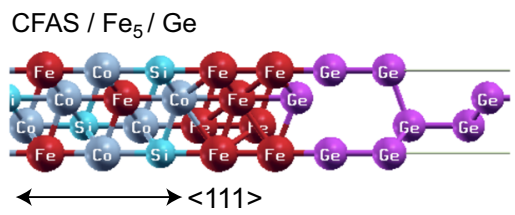

c

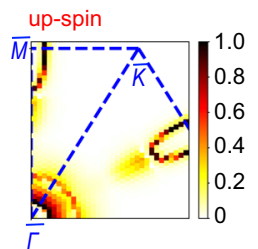

d

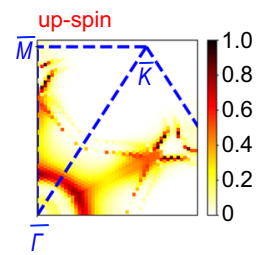

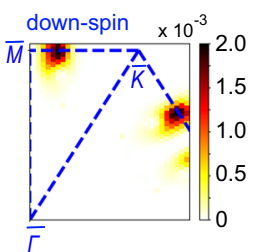

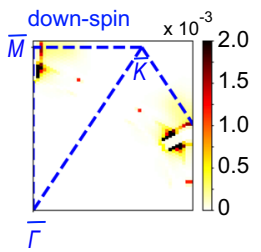

Fig. 5 Calculated transmittance of the spin-resolved conductance. $\mathbf{a}$, b Theoretically optimized interfaces of CFAS/Ge and CFAS/Fe $5 / G e$, respectively, for the heterojunctions considered in DFT calculations. $\mathbf{c}, \mathbf{d}$ k-point-resolved transmittances of up-spin and down-spin electrons for $\mathrm{CFAS} / \mathrm{Ge}$ and $\mathrm{CFAS} / \mathrm{Fe}_{5} / \mathrm{Ge}$, respectively.

In the experiment, although the used CFAS layer was not perfectly half-metallic and the performance of the CFAS/Ge direct junctions was still poor due to the degradation of the interface quality ${ }^{20}$, the insertion of $\mathrm{Fe}_{5-6}$ between CFAS and Ge contributed to both the improvement in the crystal quality of the CFAS layer and the promotion of the $\Lambda_{1}$ band symmetry matching for highly spin-polarized current flows. In addition, the spin polarization could not be enhanced by the symmetry matching of electronic bands between $\mathrm{Fe}(111)$ and $\mathrm{Ge}$ $(111)^{19}$ because there were bands with the same $\Lambda_{1}$ band symmetry at the Fermi level in both spin channels, as shown in Figs. 4b and c. Furthermore, we already verified that the band structure of the B2-ordered CFAS maintains the energy-band symmetry matching and Fermi-surface asymmetry for the efficient spin injection considered here. From these considerations, an architecture of spin injection through energy-band symmetry matching between half-metallic CFAS and Ge along the $<111>$ direction, the atomically controlled Fe layers can be considered for demonstrating highly efficient spin transport in Ge.

We have shown a one-order enhancement in the spin signals in Ge-based LSV devices with $\mathrm{Fe}_{5-6}$, which can be interpreted in terms of the spin injection through the energy-band symmetry matching at the $\mathrm{CFAS} / \mathrm{Fe}_{5-6} / \mathrm{Ge}$ electrodes along the $<111>$ direction. From the results in Figs. $1 \mathrm{~b}$ and 2 , as the number of inserted $\mathrm{Fe}$ atomic layers was increased to more than seven-ten, the values of | $\Delta R_{\mathrm{NL}}|,| \Delta R_{\mathrm{L}} \mid, P$, and $\gamma$ gradually decreased. When the spin-dependent transmittance and spin polarization for the $\mathrm{CFAS} / \mathrm{Fe}_{9} / \mathrm{Ge}$ heterointerface were also calculated by the DFT calculations, the spin-dependent transmittance $G_{\text {up }}$ and $G_{\text {down }}$ values were $1.2 \times 10^{-1}$ and $9.1 \times 10^{-4}$ for the up-spin and down-spin electrons, respectively. Because $P_{\text {cal }} \sim 1.0$ even for CFAS $/ \mathrm{Fe}_{9} / \mathrm{Ge}$, we conclude that the increase in the thickness of the inserted $\mathrm{Fe}$ atomic layers from $\mathrm{Fe}_{5}$ to $\mathrm{Fe}_{9}$ does not theoretically affect the spin-polarized currents. Thus, there is another mechanism behind the decrease in the values of $\left|\Delta R_{\mathrm{NL}}\right|,\left|\Delta R_{\mathrm{L}}\right|, P$, and $\gamma$ after the number of inserted Fe atomic layers is increased to more than seven-ten.

Although the spin diffusion length of Fe at low temperatures is expected to be $\sim 8 \mathrm{~nm}^{33}$, much longer than the thickness of the inserted Fe atomic layers, the degradation of the spin injection/detection efficiency should be considered in actual LSV devices. That is, the present experiments include both spin injection into Ge through the energy-band symmetry matching with CFAS and diffusive spin detection from Ge into CFAS via the inserted $\mathrm{Fe}$ atomic layers. Even though efficient spin injection through energy-band symmetry matching was demonstrated at the spin injector in actual LSV devices, the values of $\left|\Delta R_{\mathrm{NL}}\right|$ and $\left|\Delta R_{\mathrm{L}}\right|$ could also be affected by the diffusive spin transport properties in the Ge channel layer and at the spin detector.

Finally, we present $\left|\Delta R_{\mathrm{NL}}\right|$ and $\left|\Delta R_{\mathrm{L}}\right|$ at room temperature $(296 \mathrm{~K})$ as a function of the number of inserted Fe atomic layers in Fig. 6a. Although the values of $\left|\Delta R_{\mathrm{NL}}\right|$ and $\left|\Delta R_{\mathrm{L}}\right|$ are relatively low compared to those in Fig. 1b because of the presence of spin relaxation in $\mathrm{Ge}^{15,16}$ and the decrease in the spin polarization of CFAS, the tendency observed here is similar to that in Fig. 1b. This means that the effect of spin injection through the energyband symmetry matching between CFAS and Ge via $\mathrm{Fe}_{5-6}$ on the spin transport in the LSV device can be observed even at room temperature. In Fig. 6b, we can clearly observe spin-valve-like hysteretic behavior two orders of magnitude larger than those reported previously in refs. ${ }^{4,17}$. Note that the room-temperature MR ratio, i.e., $\Delta R_{\mathrm{L}} / R \times 100$, for the LSV device with $\mathrm{Fe}_{5-6}$ is $\sim 0.04 \%$, which is approximately two orders of magnitude larger than those in the LSV devices without insertion of $\mathrm{Fe}$ 

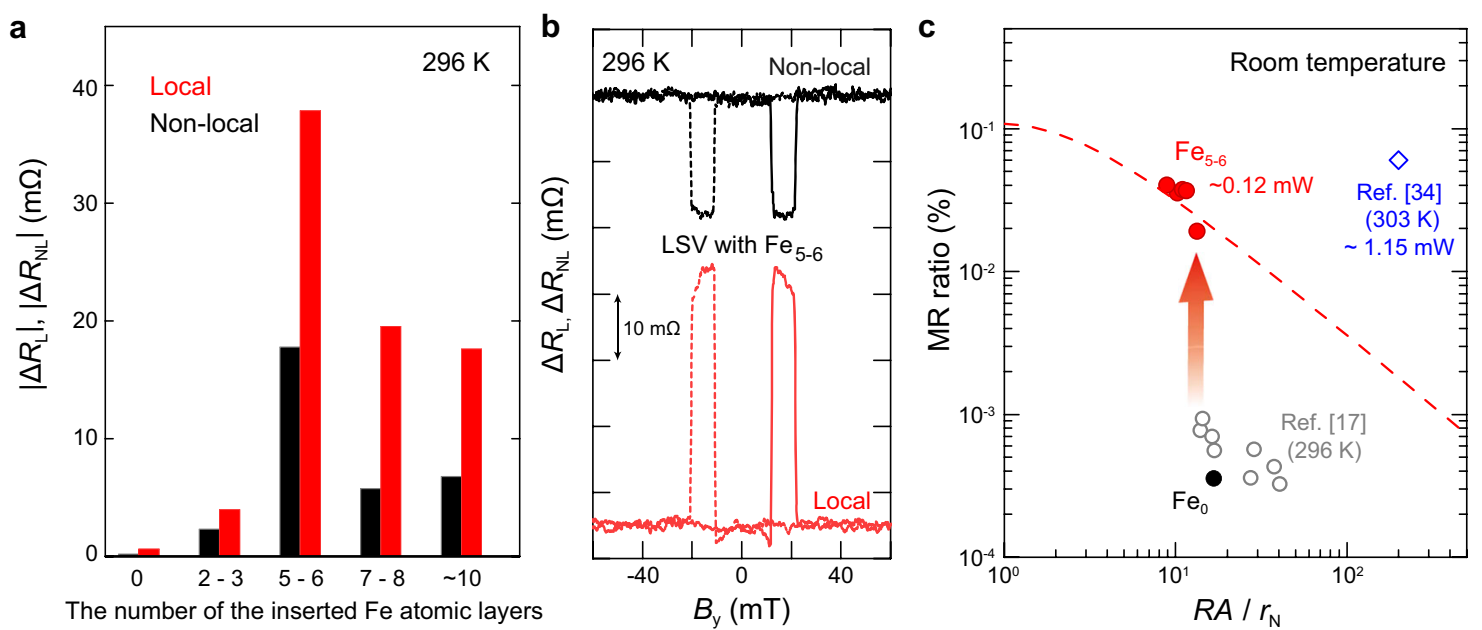

Fig. 6 Room-temperature spin transport data. a Variations in $\Delta R_{N L}$ and $\Delta R_{L}$ with the number of inserted Fe atomic layers at $296 \mathrm{~K}$. b Nonlocal and local magnetoresistance curves measured at $296 \mathrm{~K}$ and $I=-1.0 \mathrm{~mA}$ for an LSV device with $\mathrm{Fe}_{5-6}$. c Room-temperature MR ratio (\%) for LSV devices with $\mathrm{Fe}_{5-6}$ (red) and no Fe insertion $\mathrm{Fe}_{0}$ (black), together with those in ref. ${ }^{17}$ for Ge (gray) and in ref. ${ }^{34}$ for Si (blue). The electric power for obtaining the largest MR ratio is one order of magnitude lower than that in Si-based LSV devices with MgO tunnel barriers ${ }^{34}$. The value of $r_{\mathrm{N}}$ is the spin resistance of the spin-transport layers, where $r_{N}=8.69 \Omega \mu \mathrm{m}^{2}$ at $296 \mathrm{~K}$ for the $n$-Ge used here. The red dashed curve is a guide line based on the standard theory with $\gamma=0.1\left(\right.$ ref. $^{38}$ ). The value of $P$ for an LSV device with $\mathrm{Fe}_{5-6}$ is estimated to be 0.075 at room temperature from the same method as Fig. 2.

atomic layers $^{17}$ and is on the same order as the highest value at room temperature in Si-based LSV devices with $\mathrm{MgO}$ tunnel barriers ${ }^{34}$, where $R$ is the resistance value measured in the parallel magnetization states of LSV devices. A detailed comparison of the MR ratios in LSV devices between CFAS/Fe ${ }_{5-6} / \mathrm{Ge}$ Schottky-tunnel electrodes and $\mathrm{CoFe} / \mathrm{MgO} / \mathrm{Si}$ electrodes is presented in Fig. 6c. Because our LSV devices do not utilize insulators as tunnel barriers at the electrodes, the values of $R A$ at low applied bias voltages $(\sim 0.12 \mathrm{~V})$ for obtaining the highest MR ratio can be less than $0.20 \mathrm{k} \Omega \mu \mathrm{m}^{2}$ (Supplementary Fig. S1), maintaining the low parasitic resistance at the electrodes. On the other hand, for Si-based LSV devices with $\mathrm{CoFe} / \mathrm{MgO} / \mathrm{Si}$ electrodes, quite high bias voltages $(\sim 2.3 \mathrm{~V})$ should be required for obtaining the largest MR ratio at room temperature. Due to this superiority, we can reduce the electric power for obtaining the largest MR ratio at room temperature down to $\sim 0.12 \mathrm{~mW}$, one order of magnitude lower than that $(\sim 1.15 \mathrm{~mW})$ in Si-based LSV devices with $\mathrm{MgO}$ tunnel barriers ${ }^{34}$. Therefore, spin injection technique with a band symmetry-matched CFAS/Fe/Ge(111) heterostructure is available for achieving high-performance SC-based spintronic devices even at room temperature. If energy-band symmetry matching without the use of insulator barrier layers is proposed and developed for other semiconducting channel materials, such as $\mathrm{Si}^{3,7,34}$ and graphene ${ }^{35,36}$, then efficient spin injection techniques can be utilized even for other group IV SC spintronic devices that can operate at room temperature. This approach provides a new solution for the simultaneous achievement of a high MR ratio and a low parasitic resistance in future SC spintronic architectures at room temperature.

In conclusion, we have shown an efficient spin injection technique for an SC using an atomically controlled ferromagnet/ferromagnet/SC heterostructure with lowresistive Schottky-tunnel barriers. On the basis of symmetry matching of the electronic bands between the top highly spin-polarized ferromagnet and the SC, the magnitude of spin signals in LSV devices can be enhanced by up to one order of magnitude compared to those obtained with conventional ferromagnet/SC structures. Because the spin injection technique with energy-band symmetry matching between the highly spin-polarized ferromagnet and the SC presented here did not require the use of insulator tunnel barriers at the ferromagnet/SC electrodes in the device structures, we simultaneously achieved the best device performance, i.e., the same order of magnitude as the highest MR ratio at room temperature and the lowest electric power at the electrodes. This approach provides a new solution for the simultaneous achievement of highly efficient spin injection and low electric power at the electrodes in SC devices, leading to novel SC spintronic architectures at room temperature.

\section{Materials and methods}

\section{Growth of FM/SC layers and device fabrication}

First, we grew an undoped $\mathrm{Ge}(111)$ layer $(\sim 28 \mathrm{~nm})$ at $350^{\circ} \mathrm{C}$ (LT-Ge) on a commercial undoped $\mathrm{Si}(111)$ substrate $(\rho \sim 1000 \Omega \mathrm{cm})$, followed by an undoped $\mathrm{Ge}(111)$ 
layer $(\sim 70 \mathrm{~nm})$ grown at $700{ }^{\circ} \mathrm{C}(\mathrm{HT}-\mathrm{Ge})$ by molecular beam epitaxy $(\mathrm{MBE})^{16}$. Then, a 140-nm-thick phosphorus (P)-doped $n^{+}$-Ge(111) layer (doping concentration $\sim 10^{19} \mathrm{~cm}^{-3}$ ) was grown by MBE at $350{ }^{\circ} \mathrm{C}$ on top of it as a spin transport layer. The electron carrier concentration was estimated to be $6.0-7.0 \times 10^{18} \mathrm{~cm}^{-3}$. From the free electron model, the position of the Fermi level in $n$-Ge is roughly estimated to be $\sim 78.6 \mathrm{meV}$ above the conduction band edge. To promote tunneling conduction at FM/SC Schottky interfaces, two $\mathrm{P} \delta$ doped Ge layers with an ultrathin Si insertion layer were grown on top of the $n^{+}$-Ge layer ${ }^{16}$. As a spin injector and detector, double FM/FM layer structures were fabricated as follows. First, we controlled the deposition by MBE below $80^{\circ} \mathrm{C}$ of the Fe atomic layer (AL) from 0 to $\sim 10 \mathrm{AL}$ on the $\delta$-doped Ge layers. Next, we grew a Heusler alloy, $\mathrm{Co}_{2} \mathrm{FeAl}_{0.5} \mathrm{Si}_{0.5}$ (CFAS), with a thickness of $8 \mathrm{~nm}$ on top of it by using nonstoichiometric growth techniques with Knudsen cells during $\mathrm{MBE}^{15,16}$. As a result, Schottky tunnel conduction of electrons for electrical spin injection from CFAS into Ge through the controlled Fe atomic layers was demonstrated. Finally, the grown layers were patterned into electrodes with sizes of $0.4 \times 5.0 \mu \mathrm{m}^{2}$ (FM1) and $0.5 \times 5.0 \mu \mathrm{m}^{2}$ (FM2). Note that since the two P $\delta$-doped layers were removed in the spin-transport channel layer, the spin transport shown in this article was not influenced by the $\mathrm{P} \delta$ doped layers. The edge-to-edge distance $(d)$ between the $\mathrm{FM} / \mathrm{n}$-Ge electrodes was designed to be $\sim 0.45 \mu \mathrm{m}$. Schematic aspects of the fabricated devices were shown in our previous works ${ }^{15-17,20}$. For all the LSV devices in this paper, the size of FM contacts and the width and thickness of SC layers were the same.

\section{Measurements and data analyses}

By applying in-plane magnetic fields $\left(B_{y}\right)$, nonlocal and local MR measurements were carried out in the two- and four-terminal schemes, respectively, with a standard dc method $^{23-26}$, leading to $\Delta R_{\mathrm{NL}}$ versus $B_{y}$ or $\Delta R_{\mathrm{L}}$ versus $B_{y}$ curves at various temperatures. We also applied out-ofplane magnetic fields $\left(B_{z}\right)$ under parallel and antiparallel magnetization configurations to record $\Delta R_{\mathrm{NL}}$ as a function of $B_{z}$. To roughly estimate the spin injection/detection efficiency $(P)$ at the spin injector and spin detector contacts from $\Delta R_{\mathrm{NL}}$, we used the following relation ${ }^{1,23,24}$ :

$$
\frac{\Delta V_{\mathrm{NL}}}{I}=\Delta R_{\mathrm{NL}}=\frac{P^{2} \rho_{\mathrm{N}} \lambda_{\mathrm{N}}}{S} \exp \left(-\frac{d}{\lambda_{\mathrm{N}}}\right),
$$

where $\rho_{\mathrm{N}}$ and $S$ are the resistivity $\left(1.59 \mathrm{~m} \Omega \mathrm{cm} \leq \rho_{\mathrm{N}} \leq\right.$ $2.16 \mathrm{~m} \Omega \mathrm{cm})$ and the cross-sectional area $\left(0.98 \mu \mathrm{m}^{2}\right)$ of the SC layer. For our Ge spin transport layer, the value of $\lambda_{\mathrm{N}}$ was already clarified to be $\lambda_{\mathrm{N}}=0.84 \pm 0.07 \mu \mathrm{m}$ at $8 \mathrm{~K}$ (Supplementary Fig. S2), nearly consistent with those in the previous work $^{37}$. Although there is a bias-current dependence of $\Delta R_{\mathrm{L}}$, in this article, we used the highest value of $\Delta R_{\mathrm{L}}$ at a low bias $I$ at $8 \mathrm{~K}$. We can also estimate the interface spin polarization $\gamma$ at the spin injector and spin detector contacts from $\Delta R_{\mathrm{L}}$. In general, the magnitude of $\Delta R_{\mathrm{L}}$ in an FM/SC/FM structure with double tunnel barriers has been expressed as follows ${ }^{6,38}$ :

$$
\frac{\Delta V_{\mathrm{L}}}{I}=\Delta R_{\mathrm{L}}=\frac{8 \gamma_{1} \gamma_{2} r_{\mathrm{b}}^{* 2} r_{\mathrm{N}}}{\left\{\left(2 r_{\mathrm{b}}^{*}+r_{\mathrm{N}}\right)^{2} \exp \left(\frac{d}{\lambda_{\mathrm{N}}}\right)-r_{\mathrm{N}}^{2} \exp \left(-\frac{d}{\lambda_{\mathrm{N}}}\right)\right\} S},
$$

where $\gamma_{1}$ and $\gamma_{2}$ are the spin polarizations of the FM1/SC and FM2/SC interfaces, $r_{\mathrm{b}}{ }^{*}$ indicates the RA value for the $\mathrm{FM} / \mathrm{SC}$ interfaces, and $r_{\mathrm{N}}$ is the spin resistance of the SC layer.

\section{Computational details for calculations}

We performed theoretical calculations on the basis of first-principles density functional theory (DFT) using the Quantum-ESPRESSO package ${ }^{39}$ with ultrasoft pseudopotentials and a plane-wave basis set. The cutoff energies for the wave function and charge density were set to 40 and $400 \mathrm{Ry}$, respectively. To evaluate the transmittance of the spin-resolved conductance, we used the PWCOND program package ${ }^{40}$, implemented in Quantum-ESPRESSO. We considered an open quantum system consisting of a scattering region corresponding to a 111-oriented CFAS/ $\mathrm{Fe} / \mathrm{Ge} / \mathrm{Fe} / \mathrm{CFAS}$ heterostructure attached to left and right semi-infinite electrodes corresponding to bulk CFAS with a hexagonal unit cell. The transmittance was obtained from scattering equations with infinite boundary conditions in which the wave function of the scattering region and the derivative were smoothly connected to the Bloch states of each electrode. The CFAS/Ge/CFAS heterojunction with Fe surface termination of CFAS was constructed by 49 CFAS and 16 Ge atomic layers. The CFAS/ $\mathrm{Fe}_{5} / \mathrm{Ge} / \mathrm{Fe}_{5} / \mathrm{CFAS}$ heterojunction, where $\mathrm{Fe}_{5}$ means 5 atomic layers of Fe, was constructed by $10 \mathrm{Fe}, 39 \mathrm{CFAS}$, and $16 \mathrm{Ge}$ atomic layers.

The structures of these heterojunctions were optimized while keeping the in-plane lattice constant at $4.07 \AA$, which is the calculated equilibrium lattice constant of diamond Ge with a hexagonal unit cell. Here, $12 \times 12 \times 1$ and $96 \times 96 k$-meshes were adopted to obtain the electronic structures and optimized structure of the scattering region and the $k$-point-averaged transmittance, respectively. The nonstoichiometric $\mathrm{Al}_{0.5} \mathrm{Si}_{0.5}$ sites were treated by using the virtual crystal approximation. For all calculations, the Methfessel-Paxton smearing method with a broadening parameter of $0.01 \mathrm{Ry}$ was used, and the $\mathrm{GGA}^{30}$ was applied for the exchange and correlation functional. 


\section{Acknowledgements}

This work was financially supported by Grants-in-Aid for Scientific Research (A) and (S) (No. 16H02333, 17H06120, and 19H05616) from the Japan Society for the Promotion of Science (JSPS). M.Y. acknowledges JSPS Research Fellowships for Young Scientists (No. 18J00502).

\section{Author details}

${ }^{1}$ Center for Spintronics Research Network, Graduate School of Engineering Science, Osaka University, 1-3 Machikaneyama, Toyonaka 560-8531, Japan. ${ }^{2}$ Department of Material Engineering Science, Graduate School of Engineering Science, Osaka University, 1-3 Machikaneyama, Toyonaka 560-8531, Japan. ${ }^{3}$ Institute for NanoScience Design, Osaka University, Toyonaka 560-8531, Japan. ${ }^{4}$ Institute for Datability Science, Osaka University, Suita 565-0871, Japan. ${ }^{5}$ Advanced Research Laboratories, Tokyo City University, 8-15-1 Todoroki, Tokyo 158-0082, Japan. ${ }^{6}$ Institute of Scientific and Industrial Research, Osaka University, Ibaraki 567-0047, Japan. ${ }^{7} \mathrm{MaDIS}$, National Institute for Materials Science, Tsukuba 305-0047, Japan

\section{Author contributions}

M.Y. and K.H. proposed and supervised this study. The growth of ferromagnetic and semiconductor layers was conducted by M.Y. and S.Y. and by M.Y. and K.S., respectively. K.H. and M.Y. designed the lateral spin device structures fabricated by M.Y. and M.T. Spin transport measurements and analyses were also performed by M.Y. and M.T. Theoretical calculations were carried out by F.K. and T.F., and the related data analyses were performed by F.K, T.F., and T.O. All authors contributed to the discussion and interpretation of the results and preparation of the manuscript.

\section{Conflict of interest}

The authors declare that they have no conflict of interest.

\section{Publisher's note}

Springer Nature remains neutral with regard to jurisdictional claims in published maps and institutional affiliations.

Supplementary information is available for this paper at https://doi.org/ 10.1038/s41427-020-0228-5.

Received: 27 December 2019 Revised: 27 April 2020 Accepted: 27 April 2020.

Published online: 19 June 2020

\section{References}

1. Lou, X. et al. Electrical detection of spin transport in lateral ferromagnet-semiconductor devices. Nat. Phys. 3, 197-202 (2007).

2. Appelbaum, I., Huang, B. \& Monsma, D. J. Electronic measurement and control of spin transport in silicon. Nature 447, 295-298 (2007).

3. Suzuki, T. et al. Room-temperature electron spin transport in a highly doped $\mathrm{Si}$ channel. Appl. Phys. Express 4, 023003 (2011).

4. Yamada, M. et al. Room-temperature spin transport in $n$-Ge probed by fourterminal nonlocal measurements. Appl. Phys. Express 10, 093001 (2017).

5. Rashba, E. I. Theory of electrical spin injection: tTunnel contacts as a solution of the conductivity mismatch problem. Phys. Rev. B 62, R16267-R16270 (2000).

6. Fert, A. \& Jaffrès, H. Conditions for efficient spin injection from a ferromagnetic metal into a semiconductor. Phys. Rev. B 64, 184420 (2001).

7. Spiesser, A. et al. Giant spin accumulation in silicon nonlocal spin-transport devices. Phys. Rev. Appl. 8, 064023 (2017).

8. Spiesser, A., Saito, H., Yuasa, S. \& Jansen, R. Tunnel spin polarization of Fe/MgO/ Si contacts reaching $90 \%$ with increasing MgO thickness. Phys. Rev. B 99, 224427 (2019).

9. Tanamoto, T. et al. Scalability of spin field programmable gate array: a reconfigurable architecture based on spin metal-oxide-semiconductor field effect transistor. J. Appl. Phys. 109, 07C312 (2011).

10. Dery, H., Dalal, P., Cywiński, Ł. \& Sham, L. J. Spin-based logic in semiconductors for reconfigurable large-scale circuits. Nature 447, 573-576 (2007).

11. Tanaka, M. \& Sugahara, S. MOS-based spin devices for reconfigurable logic. IEEE Trans. Electron Dev. 54, 961-976 (2007).

12. Koo, H. C. et al. Control of spin precession in a spin-injected field effect transistor. Science 325, 1515-1518 (2009).
13. Cesari, S. D., Bergamaschini, R., Vitiello, E., Giorgioni, A. \& Pezzoli, F. Optically reconfigurable polarized emission in germanium. Sci. Rep. 8, 11119 (2018).

14. Watzinger, $\mathrm{H}$. et al. A germanium hole spin qubit. Nat. Commun. 9, 3902 (2018).

15. Fujita, Y. et al. Spin transport and relaxation up to $250 \mathrm{~K}$ in heavily doped $n$ type Ge detected using $\mathrm{Co}_{2} \mathrm{FeAl}_{05} \mathrm{Si}_{05}$ electrodes. Phys. Rev. Appl. 8, 014007 (2017).

16. Hamaya, K. et al. Spin transport and relaxation in germanium. J. Phys. D Appl. Phys. 51, 393001 (2018).

17. Tsukahara, M. et al. Room-temperature local magnetoresistance effect in $n$-Ge devices with low-resistive Schottky-tunnel contacts. Appl. Phys. Express 12 033002 (2019).

18. Mavropoulos, P., Wunnicke, O. \& Dederichs, P. H. Ballistic spin injection and detection in Fe/semiconductor/Fe junctions. Phys. Rev. B 66, 024416 (2002).

19. Wunnicke, O., Mavropoulos, P., Zeller, R. \& Dederichs, P. H. Ballistic spin injection from Fe into ZnSe(001), (111), and (110), and into GaAs(001). J. Phys. Condens. Matter 16, 4643-4659 (2004).

20. Kuerbanjiang, B. et al. Correlation between spin transport signal and Heusler/ semiconductor interface quality in lateral spin-valve devices. Phys. Rev. B 98, 115304 (2018).

21. Fujita, $Y$. et al. Nonmonotonic bias dependence of local spin accumulation signals in ferromagnet/semiconductor lateral spin-valve devices. Phys. Rev. B 100, 024431 (2019)

22. Shan, R. et al. Demonstration of half-metallicity in Fermi-level-tuned Heusler alloy $\mathrm{CO}_{2} \mathrm{FeAl}_{0.5} \mathrm{Si}_{0.5}$ at room temperature. Phys. Rev. Lett. 102, 246601 (2009).

23. Johnson, M. \& Silsbee, R. H. Interfacial charge-spin coupling: Injection and detection of spin magnetization in metals. Phys. Rev. Lett. 55, 1790-1793 (1985).

24. Jedema, F. J., Heersche, H. B., Filip, A. T., Baselmans, J. J. A. \& van Wees, B. J. Electrical detection of spin precession in a metallic mesoscopic spin valve. Nature 416, 713-716 (2002).

25. Jedema, F. J., Nijboer, M. S., Filip, A. T. \& van Wees, B. J. Spin injection and spin accumulation in all-metal mesoscopic spin valves. Phys. Rev. B 67, 085319 (2003).

26. Kimura, T., Hashimoto, N., Yamada, S., Miyao, M. \& Hamaya, K. Roomtemperature generation of giant pure spin currents using epitaxial $\mathrm{Co}_{2} \mathrm{FeSi}$ spin injectors. NPG Asia Mater. 4, e9 (2012).

27. Jansen, R. et al. Nonlinear electrical spin conversion in a biased ferromagnetic tunnel. Contact Phys. Rev. Appl. 10, 064050 (2018).

28. Nedelkoski, Z. et al. Realisation of magnetically and atomically abrupt halfmetal/semiconductor interface: $\mathrm{Co}_{2} \mathrm{FeAl}_{0.5 \mathrm{Si}} \mathrm{S}_{0 .} / \mathrm{Ge}(111)$. Sci. Rep. 6, 37282 (2016).

29. Oyarzún, S. et al. Evidence for spin-to-charge conversion by Rashba coupling in metallic states at the Fe/Ge(111) interface. Nat. Commun. 7, 13857 (2016).

30. Perdew, J. P., Burke, K. \& Ernzerhof, M. Generalized gradient approximation made simple. Phys. Rev. Lett. 77, 3865-3868 (1996).

31. Bulter, W. J. Zhang, X.-G., Schulthess, T. C. \& MacLaren, J. M. Spin-dependent tunneling conductance of Fe|MgO|Fe sandwiches. Phys. Rev. B 63, 054416 (2001).

32. Miura, Y., Futatsukawa, K., Nakajima, S., Abe, K. \& Shirai, M. First-principles study of ballistic transport properties in $\mathrm{CO}_{2} \mathrm{MnSi} / \mathrm{X} / \mathrm{CO}_{2} \mathrm{MnSi}(001)(\mathrm{X}=\mathrm{Ag}, \mathrm{Au}, \mathrm{Al}, \mathrm{V}$, Cr) trilayers. Phys. Rev. B 84, 134432 (2011).

33. Bass, J. \& Pratt Jr., W.P. Sp in-diffusion lengths in metals and alloys, and spinflipping at metal/metal interfaces: an experimentalist's critical review. J. Phys. Condens. Matter 19, 183201 (2007)

34. Ishikawa, M., Tsukahara, M., Yamada, M., Saito, Y. \& Hamaya, K. Local magnetoresistance at room temperature in $\mathrm{Si}<100>$ devices. IEEE Trans. Magn. 54, 1400604 (2018)

35. Han, W., Kawakami, R. K., Gmitra, M. \& Fabian, J. Graphene spintronics. Nat. Nanotechnol. 9, 794-807 (2014)

36. Dankert, A. \& Dash, S. P. Electrical gate control of spin current in van der Waals heterostructures at room temperature. Nat. Commun. 8, 16093 (2017).

37. Yamada, $\mathrm{M}$. et al. Large impact of impurity concentration on spin transport in degenerate $n$-Ge. Phys. Rev. B 95, 161304(R) (2017).

38. Jaffrès, H., George, J.M. \& Fert, A. Spin transport in multiterminal devices: large spin signals in devices with confined geometry. Phys. Rev. B 82, 140408(R) (2010).

39. Giannozzi, P. et al. QUANTUM ESPRESSO: a modular and open-source software project for quantum simulations of materials. J. Phys. Condens. Matter 21, 395502 (2009).

40. Choi, H. J. et al. Ab initio pseudopotential method for the calculation of conductance in quantum wires. Phys. Rev. B 59, 2267 (1999). 